\title{
TU/e EmonOWEN

\section{Universal model of finite Reynolds number turbulent flow in channels and pipes}

\section{Citation for published version (APA):}

L'vov, V. S., Procaccia, I., \& Rudenko, O. (2008). Universal model of finite Reynolds number turbulent flow in channels and pipes. Physical Review Letters, 100(5), 054504-1/4. [054504].

https://doi.org/10.1103/PhysRevLett.100.054504

DOI:

10.1103/PhysRevLett.100.054504

Document status and date:

Published: 01/01/2008

\section{Document Version:}

Publisher's PDF, also known as Version of Record (includes final page, issue and volume numbers)

\section{Please check the document version of this publication:}

- A submitted manuscript is the version of the article upon submission and before peer-review. There can be important differences between the submitted version and the official published version of record. People interested in the research are advised to contact the author for the final version of the publication, or visit the $\mathrm{DOI}$ to the publisher's website.

- The final author version and the galley proof are versions of the publication after peer review.

- The final published version features the final layout of the paper including the volume, issue and page numbers.

Link to publication

\section{General rights}

Copyright and moral rights for the publications made accessible in the public portal are retained by the authors and/or other copyright owners and it is a condition of accessing publications that users recognise and abide by the legal requirements associated with these rights.

- Users may download and print one copy of any publication from the public portal for the purpose of private study or research.

- You may not further distribute the material or use it for any profit-making activity or commercial gain

- You may freely distribute the URL identifying the publication in the public portal.

If the publication is distributed under the terms of Article 25fa of the Dutch Copyright Act, indicated by the "Taverne" license above, please follow below link for the End User Agreement:

www.tue.nl/taverne

Take down policy

If you believe that this document breaches copyright please contact us at:

openaccess@tue.nl

providing details and we will investigate your claim. 


\title{
Universal Model of Finite Reynolds Number Turbulent Flow in Channels and Pipes
}

\author{
Victor S. L'vov, Itamar Procaccia, and Oleksii Rudenko \\ Department of Chemical Physics, The Weizmann Institute of Science, Rehovot 76100, Israel
}

(Received 30 May 2007; published 8 February 2008)

\begin{abstract}
In this Letter, we suggest a simple and physically transparent analytical model of pressure driven turbulent wall-bounded flows at high but finite Reynolds numbers Re. The model provides an accurate quantitative description of the profiles of the mean-velocity and Reynolds stresses (second order correlations of velocity fluctuations) throughout the entire channel or pipe, for a wide range of Re, using only three Re-independent parameters. The model sheds light on the long-standing controversy between supporters of the century-old log-law theory of von Kàrmàn and Prandtl and proposers of a newer theory promoting power laws to describe the intermediate region of the mean velocity profile.
\end{abstract}

An important challenge in wall-bounded Newtonian turbulence is the description of the profiles of the mean velocity and second order correlation functions of turbulent-velocity fluctuations throughout the entire channel or pipe at relatively high but finite Reynolds numbers. To understand the issue, focus on a channel of width $2 L$ between its parallel walls, where the incompressible fluid velocity $\boldsymbol{U}(\boldsymbol{r}, t)$ is decomposed into its average (over time) and a fluctuating part

$$
\boldsymbol{U}(\boldsymbol{r}, t)=\boldsymbol{V}(\boldsymbol{r})+\boldsymbol{u}(\boldsymbol{r}, t), \quad \boldsymbol{V}(\boldsymbol{r}) \equiv\langle\boldsymbol{U}(\boldsymbol{r}, t)\rangle .
$$

In a stationary plane channel flow with a constant pressure gradient $p^{\prime} \equiv-\partial p / \partial x$, the only component of the mean velocity $\boldsymbol{V}$ is the streamwise component $V_{x} \equiv V$ that depends on wall normal direction $z$ only. Near the wall, the mean velocity profiles for different Reynolds numbers exhibit data collapse once presented in wall units, where the Reynolds number $\operatorname{Re}_{\tau}$, the normalized distance from the wall $z^{+}$, and the normalized mean velocity $V^{+}\left(z^{+}\right)$are defined (for channels) by

$$
\operatorname{Re}_{\tau} \equiv L \sqrt{p^{\prime} L} / \nu, \quad z^{+} \equiv z \operatorname{Re}_{\tau} / L, \quad V^{+} \equiv V / \sqrt{p^{\prime} L} .
$$

The classical theory of Prandtl and von Kàrmàn for infinitely large $\operatorname{Re}_{\tau}$ is based on the assumption that the single characteristic scale in the problem is proportional to the distance from the (nearest) wall. It leads to the celebrated von Kàrmàn log-law [1]

$$
V^{+}\left(z^{+}\right)=\kappa^{-1} \ln \left(z^{+}\right)+B,
$$

which serves as a basis for the parametrization of turbulent flows near a wall in many engineering applications. On the face of it, this law agrees with the data (see, e.g., Fig. 1) for relatively large $z^{+}$, say for $z^{+}>100$, giving $\kappa \sim 0.4$ and $B \sim 5$. The range of validly of the log-law is definitely restricted by the requirement $\zeta \ll 1$, where $\zeta \equiv z / L$ (channel) ore $\zeta \equiv r / R$ (pipe of radius $R$ ). For $\zeta \sim 1$, the global geometry becomes important leading to unavoidable deviations of $V^{+}(\zeta)$ from the log-law (1), known as the wake.
The problem is that for finite $\mathrm{Re}_{\tau}$, the corrections to the $\log$-law (1) are in powers of $\varepsilon \equiv 1 / \ln \mathrm{Re}_{\tau}$ [2] and definitely cannot be neglected for the currently largest available direct numerical simulation (DNS) of channel flows $\left(\operatorname{Re}_{\tau}=2003\right.$ [3], giving $\left.\varepsilon \approx 0.13\right)$. Even for $\operatorname{Re}_{\tau}$ approaching 500000 as in the Princeton Superpipe experiment [4], $\varepsilon \approx 0.08$. This opens a Pandora's box with various possibilities to revise the log-law (1) and to replace it, as was suggested in [2], by a power law

$$
V^{+}\left(z^{+}\right)=C\left(\operatorname{Re}_{\tau}\right)\left(z^{+}\right)^{\gamma\left(\operatorname{Re}_{\tau}\right)}
$$

Here, both $C\left(\operatorname{Re}_{\tau}\right)$ and $\gamma\left(\operatorname{Re}_{\tau}\right)$ were represented as asymptotic series expansions in $\varepsilon$. The relative complexity of this proposition compared to the simplicity of Eq. (1) resulted in a less than enthusiastic response in the fluid mechanics community [5], leading to a rather fierce controversy between the log-law camp and the power-law camp. Various attempts $[2,4-8]$ to validate the log-law (1) or the alternative power law (2) were based on extensive analysis of experimental data used to fit the velocity profiles as a formal expansion in inverse powers of $\varepsilon$ or as composite expansions in both $z^{+}$and $\zeta$. Note however that in the excellent fits presented, say in [8], one uses four adjustable parameters for each function.

In this Letter, we propose a complementary approach to this issue which will finally use only three $\mathrm{Re}_{\tau}$-independent universal parameters which are the same for all the functions discussed. First, we ask what could be missed in the textbook derivations of the classical log-law (1) which may lead to different velocity profile [including possibly the power law (2)]? Our answer is: the mean turbulent velocity profile in the entire channel or pipe can be described within the traditional approach if one realizes how the characteristic length scale, which has physical meaning of the size of energy containing eddies $\ell$, depends on the position in the flow. Simple scaling near the wall, $\ell^{+}=\kappa z^{+}$, leads to the log-law (1). The alternative suggestion of [2], $\ell^{+} \propto\left(z^{+}\right)^{\alpha\left(\mathrm{Re}_{\tau}\right)}$, leads to alternative power law (2). We see no physical reason why $\ell$ should 

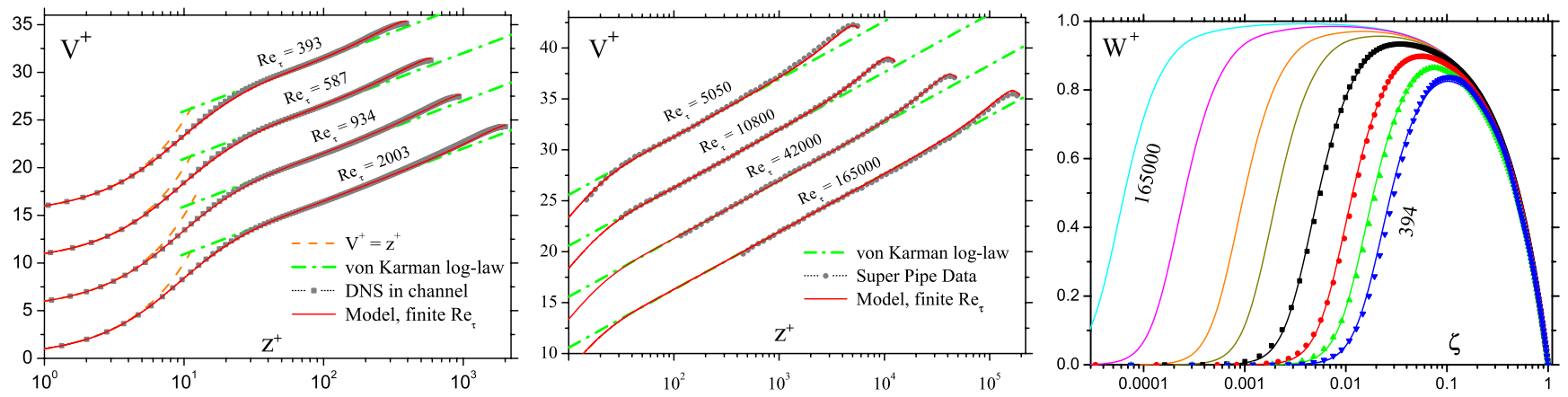

FIG. 1 (color online). Left and Middle panels: comparison of the theoretical mean velocity profiles (solid lines) at different values of $\operatorname{Re}_{\tau}$ with the DNS data for the channel flow [3,14] (Left panel, gray squares; model with $\ell_{\text {buf }}=49, \kappa=0.415, \ell_{s}=0.311$ ) and with the experimental Super-Pipe data [4] (Middle panel, gray circles; model with $\ell_{\text {buf }}=46, \kappa=0.405, \ell_{s}=0.275$ ). In dashed line, we plot the viscous solution $V^{+}=z^{+}$. In dashed-dotted line, we present the von Kàrmàn log-law. Note that the theoretical predictions with three $\mathrm{Re}_{\tau}$-independent parameters fits the data throughout the channel and pipe, from the viscous scale, through the buffer layer, the log-layer, and the wake. For clarity, the plots are shifted vertically by five units. Right panel: The Reynolds-stress profiles (solid lines) at $\operatorname{Re}_{\tau}$ from 394 to 2003 (in channel) and from 5050 to 165000 (in pipe) in comparison with available DNS data (dots) for the channel.

behave in either manner. Instead, we propose that the eddy size $\ell$ should be about $z$ for $z \ll L$, and saturate at some level $\ell_{s} \lesssim L$ approaching the center line, where the effect of the opposite wall is felt. Our analysis of DNS data provides a strong support to this idea, allowing us to get, within the traditional (second-order) closure procedure, a quantitative description of the mean shear, $S(z)=$ $d V(z) / d z$, the kinetic energy density (per unit mass), $K(z) \equiv\left\langle|\boldsymbol{u}|^{2}\right\rangle / 2$, and the tangential Reynolds stress, $W(z) \equiv-\left\langle u_{x} u_{z}\right\rangle$, in the entire flow and in a wide region of $\operatorname{Re}_{\tau}$, using only three $\operatorname{Re}_{\tau}$-independent parameters, $\kappa, B$, and $\ell_{s}\left(\ell_{s} \approx 0.311 L\right.$ for the channel and $\ell_{s} \approx 0.275 L$ for the pipe).

The closure model. - should relate three objects: $S^{+}$, $K^{+}$, and $W^{+}$. The first (exact) relation between these objects follows from the Navier-Stokes equation for the mean velocity, being the mechanical balance between the momentum generated at distance $z$ from the wall, i.e., $p^{\prime}(L-z)$, and the momentum transferred to the wall by kinematic viscosity and turbulent transport. In physical and wall units it has the form

$$
\nu S+W=p^{\prime}(L-z) \Rightarrow S^{+}+W^{+}=1-\zeta, \quad \zeta / L .
$$

Already in 1877, Boussinesq attempted to close this equation by introducing the notion of turbulent viscosity $\nu_{T}$, writing $W=\nu_{T} S$ [9]. Estimating $\nu_{T}$ as $\kappa_{W} \ell_{W} \sqrt{K}$, one finishes with the closure $W^{+}=\kappa_{W} \ell_{W}^{+} \sqrt{K^{+}} S^{+}$. Here, $\ell_{W}$ is a $\zeta$-dependent characteristic scale of energy containing eddies, determining the nonlinear dissipation of $W$, and $\kappa_{W}$ is a constant introduced here for convenience. A more careful analysis of the balance equation for $W$ (see Ref. [10] and Appendix) that includes the viscous dissipation of $W$, leads to a somewhat more involved closure for $W$ in a form involving an additional universal, $\operatorname{Re}_{\tau}$-independent dimensionless function of $z^{+}$:

$$
r_{W} W^{+} \approx \kappa_{W} \ell_{W}^{+} \sqrt{K^{+}} S^{+}, \quad r_{W}\left(z^{+}\right) \equiv\left(1+\frac{\ell_{\mathrm{buf}}^{+6}}{z^{+6}}\right)^{1 / 6} .
$$

Here, $\ell_{\text {buf }}^{+} \approx 49$ is a $\operatorname{Re}_{\tau}$-independent length that plays a role of the crossover scale (in wall units) between the buffer and log-law region. In this form, $\ell_{W}(\zeta) \propto z$ near the wall, and the choice $\kappa_{W} \approx 0.20$ ensures that $\lim _{\zeta \rightarrow 0} \ell_{W}(\zeta)=\zeta$.

A third relation to supplement Eqs. (3) and (4) is obtained by balancing the turbulent energy generated by the mean flow at a rate $S W$ and the dissipation at a rate $\varepsilon_{K} \equiv$ $\nu\left\langle|\nabla u|^{2}\right\rangle$ :

$$
S^{+} W^{+} \approx \varepsilon_{K}^{+} ; \quad \varepsilon_{K}^{+}=K^{+3 / 2} /\left[\kappa_{K} \ell_{K}^{+}\right] .
$$

Here, the dissipation is estimated via the energy cascade over scales involving a characteristic scale of energy containing eddies, $\ell_{K}(z)$ determining the energy transfer rate. The constant $\kappa_{K} \approx 3.7$ will be used to ensure that the slope of this function at $z^{+}=0$ is unity.

Note that in (4) and (5), we used a local-balance approximation, neglecting the spatial energy flux. This approximation is very good in the log-law region, but it deteriorates near the wall and near the center line. Nevertheless, for our purposes, this has no consequences. Near the wall, $W^{+} \ll S^{+}$and the local-balance approximation play no role in the exact mechanical balance (3) that determines $S$. For the same reason, we also do not need to introduce a correction $r_{K}\left(z^{+}\right)$in (5) due to the direct viscous dissipation [similar to $r_{W}\left(z^{+}\right)$in Eq. (4)] since the length scale replacing $\ell_{\text {buf }}^{+}$here will be the dissipative scale $\ell_{\text {diss }} \approx 5$ which is entirely buried in the region where $W$ and $K$ are small. Near the center line, $S^{+}$tends to zero, and Eq. (3) determines $W^{+} \approx 1-\zeta$, which allows an accurate determination of $S^{+}$, because we know that $\ell_{W}$ and $\ell_{K}$ must saturate. 
Profiles of the characteristic length scales $\ell_{K}, \ell_{W}-$ Now, we show that the source of confusion is the assumption that the relevant length scales can be determined a priori as $\ell_{K, W}^{+} \propto\left(z^{+}\right)^{\alpha}$ with $\alpha=1$ or $\alpha \neq 1$. The actual dependence $\ell_{W}$ and $\ell_{K}$ on $z$ and $L$ can be found from the data provided by the numerical simulations. Consider first $\ell_{W}$, defined by Eq. (4). We expect that plotting the scaling function $\ell_{W}^{+} / \operatorname{Re}_{\tau}$ computed for different values of $\operatorname{Re}_{\tau}$ should collapse the date onto one scaling function. The quality of the data collapse for this scaling function is presented in Fig. 2, demonstrating the expected saturation at the center line.

The second length scale, $\ell_{K}^{+}$, is determined by the second of Eq. (5). We again expect that $\ell_{K}^{+} / \operatorname{Re}_{\tau}$ should collapse the data obtained from different value of $\operatorname{Re}_{\tau}$ onto one scaling function. In Fig. 2, we demonstrate that this scaling function leads to acceptable data collapse throughout the channel and for all the four values of $\operatorname{Re}_{\tau}$ for which the simulation data are available.

Solution, velocity profiles and final scaling function.Solving Eqs. (3) together with $S^{+} W^{+}=K^{+3 / 2} /\left(\kappa_{K} \ell_{K}^{+}\right)$ that follows from Eq. (5), we find

$$
W^{+}=\left(\kappa S^{+} \ell^{+}\right)^{2} r_{W}^{-3 / 2},
$$

where we have defined the von Kàrmàn constant $\kappa \equiv$ $\left(\kappa_{W}^{3} \kappa_{K}\right)^{1 / 4} \approx 0.415$ and the crucial scaling function $\ell^{+}(\zeta)$ as follows:

$$
\ell^{+} \equiv\left[\ell_{W}^{+3}(\zeta) \ell_{K}^{+}(\zeta)\right]^{1 / 4}=\sqrt[4]{W^{+3} r_{W}^{3} / S^{+3} \varepsilon_{K}^{+}}
$$

Note that if one replaces the energy dissipation rate $\varepsilon_{K}^{+}$by the rate of energy production $W^{+} S^{+}$and takes $r_{W}$ as unity, this scaling function becomes the Prandtl mixing length [1]. However, the latter suffers from a nonphysical divergence at the center line whereas our length saturates to a constant there as it should.

The convincing data collapse for the resulting function $\ell^{+}(\zeta) / \operatorname{Re}_{\tau}$ is shown in Fig. 2, rightmost panel. Substituting Eqs. (5) and (6) in Eq. (3), we find a quadratic equation for
$S$ with a solution

$$
S^{+}=\frac{\sqrt{1+(1-\zeta)\left[2 \kappa \ell^{+}(\zeta)\right]^{2} / r_{W}\left(z^{+}\right)^{3 / 2}}-1}{2\left[\kappa \ell^{+}(\zeta)\right]^{2} / r_{W}\left(z^{+}\right)^{3 / 2}} .
$$

To integrate this equation and find the mean velocity profile for any value of $\operatorname{Re}_{\tau}$, we need to determine the scaling function $\ell^{+}(\zeta)$ from the data. A careful analysis of the DNS data allows us to find a good one-parameter fit for $\ell^{+}(\zeta)$

$$
\frac{\ell^{+}(\zeta)}{\operatorname{Re}_{\tau}}=\ell_{s}\left\{1-\exp \left[-\frac{\tilde{\zeta}}{\ell_{s}}\left(1+\frac{\tilde{\zeta}}{2 \ell_{s}}\right)\right]\right\}
$$

where $\tilde{\zeta} \equiv \zeta(1-\zeta / 2)$ and $\ell_{s} \approx 0.311$. The quality of the fit is obvious from the continuous line in the rightmost panel of Fig. 2. Note that the fit function is exactly constant at mid channel, with zero slope. This is required by symmetry, and will be the reason for our good fit of data in the wake region.

Finally, the theory for the mean velocity contains three parameters, namely $\ell_{s}$ together with $\ell_{\text {buff }}^{+}$[which determines $B$ in Eq. (1)] and $\kappa$. We demonstrate now that with these three parameters, we can determine the mean velocity profile for any value $\operatorname{Re}_{\tau}$, throughout the channel, including the viscous layer, the buffer sublayer, the loglaw region, and the wake. Examples of the integration of Eq. (8) are shown in Fig. 1. It is worthwhile to reiterate that the good fits in the viscous and the wake regions (superior to the fits presented in $[10,11]$ ), which are usually most difficult to achieve, are obtained here due to the correct asymptotics of $\ell^{+}(\zeta)$ at $\zeta \rightarrow 0$ and $\zeta \rightarrow 1$. In addition, our theory results also in the kinetic energy and Reynolds stress profiles which are in agreement with the DNS data; for $W$ profiles, see Fig. 1.

Conclusions and application to experiments. -We discussed turbulent channel flow, demonstrating the existence and usefulness of a scaling function $\ell^{+}(\zeta)$ which allows us to get the profiles of the mean velocities for all values of $\operatorname{Re}_{\tau}$ and throughout the channel, in a good agreement with
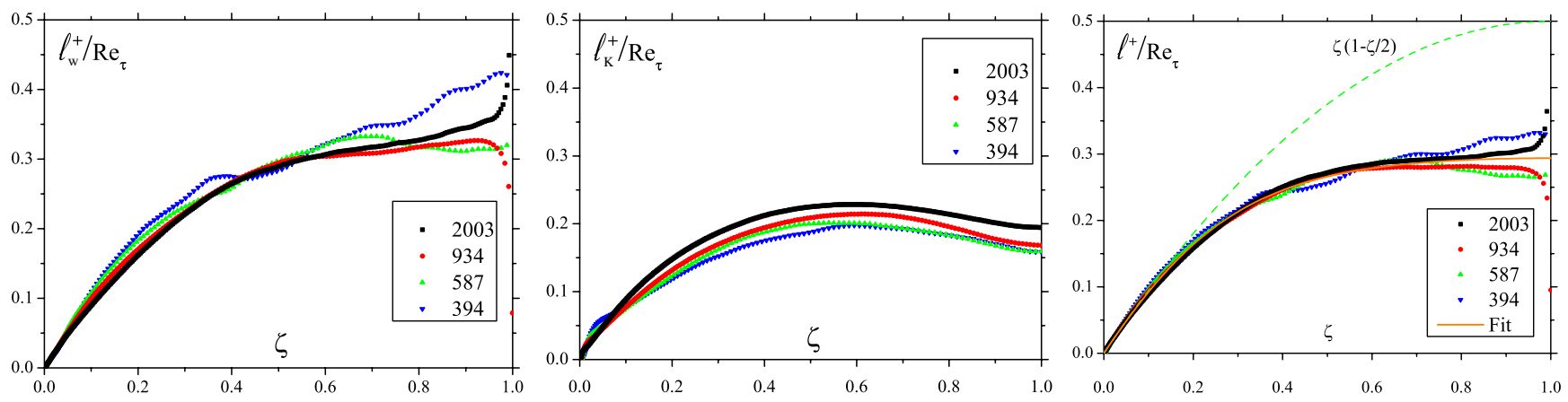

FIG. 2 (color online). The scaling function $\ell_{W}^{+}(\zeta) / \operatorname{Re}_{\tau}$ (Left panel), $\ell_{K}^{+}(\zeta) / \operatorname{Re}_{\tau}$ (Middle panel), and the final scaling function $\ell^{+}(\zeta)$ (Right panel), as a function of $\zeta \equiv z / L$, for four different values of $\operatorname{Re}_{\tau}$, computed from the DNS data [3,14]. Note the data collapse everywhere except at $\zeta \rightarrow 1$ where $W^{+} \sim S^{+} \ll 1$ and accuracy is lost. The dashed line represents $\tilde{\zeta}=\zeta(1-\zeta / 2)$ with a saturation level 0.5; in solid line, we show the fitted function Eq. (9) with $\ell_{\text {sat }}=0.311$. 
DNS. We argued that the controversy between power laws and log-laws is moot, stemming from a rough estimate of the scaling function $\ell^{+}(\zeta)$. While this function begins near the wall as $z^{+}$, it saturates later, and its full functional dependence on $\zeta$ is crucial for finding the correct mean velocity profiles. The approach also allows us to delineate the accuracy of the log-law presentation, which depends on $z^{+}$and the value of $\operatorname{Re}_{\tau}$. For asymptotically large $\operatorname{Re}_{\tau}$, the region of the log-law can be very large, but nevertheless it breaks down near the mid channel and near the buffer layer.

To show that the present approach is quite general, we apply it now to the experimental data that were at the center of the controversy [2], i.e., the Princeton University Superpipe data [4]. In Fig. 1 right panel, we show the mean velocity profiles as measured in the Superpipe compared with our prediction using the same scaling function $\ell^{+}(\zeta)$. Note that the data spans values of $\operatorname{Re}_{\tau}$ from 5050 to 165000 , and the fits with only three $\operatorname{Re}_{\tau}$-independent constants are very satisfactory. Note the $2 \%$ difference in the value of $\kappa$ between the DNS and the experimental data; we do not know at this point whether this stems from inaccuracies in the DNS or the experimental data, or whether turbulent flows in different geometries have different values of $\kappa$. While the latter is theoretically questionable, we cannot exclude this possibility until a better understanding of how to compute $\kappa$ from first principles is achieved.

We thank L. Smits for providing the data of the Princeton Superpipe and P. Monkewitz and H. Nagib for useful discussion and access to their paper prior to publication. This work is supported in part by the US-Israel Binational Science Foundation.

Appendix.-The exact balance equation for the Reynolds shear stress can be found in [1]: $P_{W}^{+}+\mathcal{R}_{W}^{+}=$ $\varepsilon_{W}^{+}-T_{W}^{+}$. Here, $P_{W}^{+}=-\tau_{y y}^{+} S^{+}$is the production of $W^{+}$, $R_{W}^{+}$is the redistribution of $W^{+}$between other Reynolds stress components, $\varepsilon_{W}^{+}$is the viscous dissipation of $W^{+}$, and $T_{W}^{+}$is the turbulent transport of $W^{+}$. Explicit expressions for these terms are in [1]. Since $\tau_{y y}$ is $\mathcal{O}(K)$, we approximate $P_{W}^{+} \propto-K^{+} S^{+} . \mathcal{R}_{W}^{+}=R_{W}^{\mathrm{RI}+}+R_{W}^{\mathrm{IP}+}[1,10]$. The first term describes the return to isotropy, while the second one is responsible for the isotropization of production. A slightly modified Rotta's model $[10,12]$ proposes that $R_{W}^{\mathrm{RI}} \propto \sqrt{K} W / \ell_{W} . R^{\mathrm{IP}}$ is modeled according to $[1,13]$, such that $R_{W}^{\mathrm{IP}} \propto K^{+} S^{+}$.

The viscous dissipation $\varepsilon W \equiv \nu\left\langle\partial_{k} u_{x} \partial_{k} u_{z}\right\rangle \quad$ is $\mathcal{O}\left(-\nu W z^{-2}\right)$. As explained in the text, we can neglect the nonlocal term $T_{W}$ in the balance for the Reynolds stress with impunity. To compensate for its loss in the viscous range, we increase the estimate $\left(-\nu W z^{-2}\right)$ by a factor $\sqrt{K / K_{*}}$, where $K_{*}$ is a dimensional constant [10] Eventually, $\varepsilon_{W}^{+} \propto-W^{+} \sqrt{K^{+}} / z^{+2}$. Hence, the approxi- mate algebraic balance equation for the Reynolds shear stress reads

$$
-a K^{+} S^{+}+b \frac{W^{+} \sqrt{K^{+}}}{\ell_{W}^{+}}+c K^{+} S^{+} \approx-d \frac{W^{+} \sqrt{K^{+}}}{z^{+2}},
$$

where $a, b, c, d$-are positive constants of $\mathcal{O}(1)$. The last equation may be rearranged to the form of the fist of Eq. (4) but with $r_{W} \equiv 1+\ell_{\text {buf }}^{+} \ell_{W}^{+} / z^{+2}, \ell_{\text {buf }}^{+} \equiv d / b$. Since the second term is dominant only near the wall where $\ell_{W}^{+}=z^{+}$, then $r_{W} \rightarrow 1+\ell_{\text {buf }}^{+} / z^{+}$. In [11], it was realized that this from, which is an interpolation between the near wall and the bulk physics, can be modeled in a way that reflects better the actual width of the buffer layer, using another interpolation formula that reads

$$
r_{W} \equiv\left[1+\left(\frac{\ell_{\text {buf }}^{+}}{z^{+}}\right)^{n}\right]^{1 / n}
$$

with $n=2$. Best fit to simulational data which is currently available is obtained with $5<n<7$. In this Letter, we chose $n=6$ leading to the second of Eqs. (4). This choice simplifies the appearance of the Eqs. (6)-(8).

[1] S. B. Pope, Turbulent Flows (Cambridge University Press, Cambridge, England, 2000), 1st ed..

[2] G. I. Barenblatt, J. Fluid Mech. 248, 513 (1993); G. I. Barenblatt and A. J. Chorin, Phys. Fluids 10, 1043 (1998).

[3] S. Hoyas and J. Jimenez, Phys. Fluids 18, 011702 (2006); DNS data at http://torroja.dmt.upm.es/ftp/channels/.

[4] B. J. McKeon, J. Li, W. Jiang, J. F. Morrison, and A. J. Smits, J. Fluid Mech. 501, 135 (2004); the data are available at http://gasdyn.princeton.edu/data/e248/mckeon_ data.html.

[5] A. J. Smits and M. V. Zagarola, Phys. Fluids 10, 1045 (1998); M. V. Zagarola, A.E. Perry, and A. J. Smits, Phys. Fluids 9, 2094 (1997).

[6] W. K. George, Phil. Trans. R. Soc. A 365, 789 (2007).

[7] R. L. Panton, Phil. Trans. R. Soc. A 365, 733 (2007).

[8] P. Monkewitz, K. A. Chauhan, and H. M. Nagib, Phys. Fluids 19, 115101 (2007).

[9] J. Boussinesq, Theorie de l'ecoulement tourbillant. (Mem. Pres. Acad. Sci., Paris, 1877), Vol. XXIII, p. 46.

[10] V. S. L'vov, I. Procaccia, and O. Rudenko, JETP Lett. 84, 67 (2006).

[11] V.S. L'vov, A. Pomyalov, I. Procaccia, and S.S. Zilitinkevich, Phys. Rev. E 73, 016303 (2006).

[12] J. C. Rotta, Z. Phys. 129, 547 (1951).

[13] D. A. Naot, A. Shavit, and M. Wolfshtein, Israel Journal of Technology 8, 259 (1970).

[14] R. G. Moser, J. Kim, and N. N. Mansour, Phys. Fluids 11, 943 (1999); DNS data at http://www.tam.uiuc.edu/ Faculty/Moser/channel. 